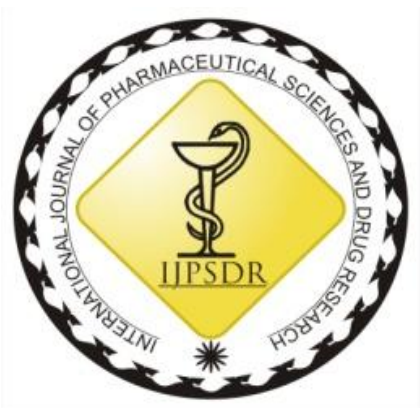

REVIEW ARTICLE

ISSN: 0975-248X CODEN (USA): IJPSPP

$($ (c) $)$ EY-NC-SA

\title{
The Impact of Physicochemical Characteristics on Therapeutic Efficacy of Anticancer Nanomaterials: A Review
}

\author{
Mensura Sied Filli1", Ahmed Abdalla Ibrahim"\#, Md Aquib', Abdul Baset Abbas3, \\ Arwa Morshed ${ }^{3}$, Kofi Oti Boakye-Yiadom¹, Samuel Kesse1, Muhammad Asim Farooq1, \\ Mily Bazezy ${ }^{1}$, Bo Wang ${ }^{*}$ \\ ${ }^{1}$ School of Pharmacy, Department of Pharmaceutics, China Pharmaceutical University, Nanjing 211198, China \\ ${ }^{2}$ School of Medicine, Department of Infectious Diseases, Xuzhou Medical University, Xuzhou 221004 China \\ ${ }^{3}$ Engineering Research Centre of Synthetic Polypeptide Drug Discovery and Evaluation, China Pharmaceutical \\ University, Nanjing 210009, China
}

Copyright (C) 2019 Mensura Sied Filli et al. This is an open access article distributed under the terms of the Creative Commons AttributionNonCommercial-ShareAlike 4.0 International License which allows others to remix, tweak, and build upon the work non-commercially, as long as the author is credited and the new creations are licensed under the identical terms.

\begin{abstract}
Cancer is a leading cause of death throughout the world which suffers from treatment failures mainly due to intensive toxicity and lack of effectiveness of conventional drugs. The application of nanotechnology in cancer treatment promises to overcome the drawbacks of conventional drugs/dosage forms and improve their therapeutic efficacy. Nanomaterials have novel properties that influence their in vivo performance. The biological behaviour of nanotechnology-based medicines in the body, which is different from the in vivo performance of conventional drug delivery systems, may provide benefits in pharmaceutical and/or clinical applications including, enhancements in solubility, stability, therapeutic efficacy, minimized side effects, and treatment of diseases. This paper discusses the unique characteristics and distinguished advantages of nanomaterials as anticancer drug carriers. Physicochemical properties of nanomaterials are critical parameters to their clinical translation. Hence, the impact of the main physicochemical properties on the efficacy of anticancer nanomaterials, which are found to effective for cancer treatment and/or diagnosis, are presented. It is important to have reliable and robust characterization techniques that could enable relate physicochemical properties of nanomaterials with their in vivo behaviour. Brief explanation of the different techniques that can be used for studying the various physicochemical characteristics of nanomaterials is given. An important consideration, to achieve fast and successful development of nanotechnologybased anticancer drug products, is assessment and optimization of physicochemical and biopharmaceutical properties at the early stage. Obviously this requires collaboration among the different discovery and development scientists.
\end{abstract}

Keywords: Nanotechnology, Cancer, Physicochemical properties, Characterization, Nanomaterials, Therapeutic efficacy. 


\section{INTRODUCTION}

Drugs play a key role in promoting a good quality of life in humans. Although many drugs that treat a wide array of diseases have reached the market, current conventional drug therapy have not overcome the limitations associated with the application of relatively bigger size materials in drug formulations, such as low bioavailability, in vivo instability, low solubility, poor gastrointestinal permeability, absence of sustained delivery and targeting to intended the site, low therapeutic efficacy, and intolerable side effects. [1] Therefore, development of new and better drugs and/carriers is required. The concept of nanotechnology has been introduced in the field of pharmacy and was applied to improve the delivery of poorly soluble drug several decades ago. It was known that small size drug particles are highly solubility in solvents, owing their large surface area. Small size liposomes, consisting of phospholipids, were first synthesized in 1960s. [2] The application of nanotechnology in medicine has been making great progresses. Various kinds of nanotechnology-based nanomaterials/drug delivery systems with variety of compositions and shapes providing different platforms to achieve many functions were developed in the past few decades. [3]

Nanomaterials exhibit unique characteristics that differ from that of bigger materials. The biological performances including, biodistribution, clearance, and toxicity of nanomaterials could differ from bigger materials owing to the unique interaction of the components of nanomaterials and their physicochemical attributes with biological organelles and molecules. ${ }^{[4]}$ Thus, nanotechnology has a great potential to be a better drug delivery carrier for conventional drugs employed for the management and drug treatment of more challenging disorders such as cancer, human immunodeficiency virus, diabetes, and hypertension. [1] Cancer is a leading cause of death globally. The therapeutic outcome of anticancer drugs is low due to their undesirable characteristics, which include poor solubility, narrow therapeutic index, and high toxicity to noncancerous cells, which may lead to treatment failure in cancer. Hence, new and better therapeutic alternatives which can deliver drug substances and other therapeutic agents which help for early detection and treatment of cancer are needed. [5] Hence, research in cancer treatment and diagnosis are the most studied fields of pharmaceutical nanotechnology. This review presents the unique characteristics and distinguished advantages of nanotechnology-based drugs/delivery systems in the treatment and/or diagnosis of cancer. The impact of physicochemical properties, which are critical to achieve successful clinical translation, on the therapeutic and/or diagnostic effectiveness of anticancer nanomaterials are discussed. Brief explanation of the different techniques that can be used for studying the different physicochemical characteristics of nanomedicines is also given.



Fig. 1: Graphical abstract

Table 1: The main unique physicochemical characteristics of anticancer nanomaterials.

\begin{tabular}{|c|c|}
\hline Parameter & Examples \\
\hline Shape & $\begin{array}{l}\text { Nanospheres, nanorods, nanovesicles, nanoshells, } \\
\text { nanotubes, nanohorns, nanohelices, nanofibers, } \\
\text { nanowires, nanoribbons, nanosticks, plate-like, etc. }\end{array}$ \\
\hline Composition & $\begin{array}{l}\text { Biological nanomaterials, organic nanomaterials, } \\
\text { inorganic nanomaterials, organi-inorganic hybrids, } \\
\text { etc. }\end{array}$ \\
\hline $\begin{array}{l}\text { Surface } \\
\text { modifications }\end{array}$ & $\begin{array}{l}\text { Polymer coat, targeting ligand, imaging contrast } \\
\text { agent }\end{array}$ \\
\hline Size & $1-100 \mathrm{~nm}$ \\
\hline Stability & $\begin{array}{l}\text { Stability in circulation, storage, and processing } \\
\text { condition }\end{array}$ \\
\hline
\end{tabular}

\section{The Unique Characteristics of Anticancer}

Nanomaterials

The unique properties of nanomaterials promise valuable benefit to cancer treatment over conventional anticancer drug formulations. To exploit the advantage that nanotechnology may offer in the treatment of cancer, it is necessary to understand the unique properties and advantages of nanomaterials. In this review the unique physicochemical characteristic are discussed. A brief explanation regarding the pharmacokinetic and theranostic properties is also presented.

Physicochemical properties affect the biological performance of drug substances. Nanomaterials have unique physicochemical properties, which include higher reactivity, small size, and large surface area, different from bigger particles of the similar components. These characteristics can be exploited to solve some of the drawbacks of conventional therapeutic and diagnostic agents. [6] The unique physical and chemical properties, which are listed in table 1 , together with the high surface area to mass ratio of nanoparticles and having similar size with biological molecules make nanomaterials a powerful tool for cancer imaging, detection and treatment. 
Therefore, in order to achieve early diagnosis and treatment of cancer researchers have been examining the role of physicochemical characteristics of a nanoparticle in its membrane permeability, distribution, clearance, toxicity and physiological effect. Since nanoparticles have a larger surface to volume ratio than macroparticles, drug loaded nanoparticles are able to release a drug faster and more effectively than bigger particles. This is of great help for low solubility and poorly absorped drugs, which is observed in a significant proportion of newly discovered and existing drugs. Along these lines, paclitaxel loaded alginate nanoparticles (PTX-ALG) synthesized for breast cancer treatment demonstrated increased solubility and enhanced antitumor effect over paclitaxel alone. [7] The high surface to volume ratios of nanomaterials enable modification with different surface functional groups that cover, encapsulate, or stabilize them. [8-9] Functionalized nanomaterials possess special functions, for instance, they may be sensitive to temperature, $\mathrm{pH}$, magnetic field, and ultrasound. [9] Hence, a polymer capped and integrin receptor targeted mesoporous silica nanoparticle (MSN) based nanocarrier (CPMSNs) was designed and synthesized for breast cancer combination therapy. The polymer shell provided $\mathrm{pH}$ sensitivity, improved biocompatibility, and facilitated functionalization of MSNs with a ligand molecule arginine-glycine-aspartic acid (cRGD) peptide. The surface modified nanocarrier CPMSN demonstrated $\mathrm{pH}$-sensitivity, cancer targeting , accumulation inside cancerous cells, controlled drug release and ability to induce apoptosis in both Triple Negative Breast Cancer (TNBC) and Multidrug resistant breast cancer cells as investigated by in vitro and in vivo preclinical studies. [10]

Moreover, nanomaterials have unique pharmacokinetic properties. Anticancer drugs that are transformed in to nano size range may have prolonged circulation, enhanced efficacy and site-specific distribution in vivo. The unique pharmacokinetics of nanomaterial can enhance their bioavailability by preventing renal clearance of drugs and protecting against clearance by the reticuloendothelial system (RES). [11] In one study, drug loaded liposomes designed to target cancer cell mitochondria were constructed in to overcome intrinsic multidrug resistance (MDR) of breast cancer and it showed higher efficacy than free drugs in vivo. It was suggested that the PEGylated liposomes escaped from the reticuloendothelial system, and accumulated more in the tumor tissues rendered by the enhanced permeability and retention effect. [12] Moreover, to enhance tumor accumulation and distribution of gold nanoparticles, a nanoplatform was designed by fabricating gold $(\mathrm{Au})$ nanorods and loading them into human induced pluripotent stem cells. The nanoplatform was found to be spatially distributed and retained long in Human gastric cancer cell lines (MGC803) tumor tissue in mice. ${ }^{[13]}$
In addition to drugs, nanomaterials can load and deliver genes and proteins in vivo. For example, a nanoparticle-stabilized nanocapsules (NPSCs) that allow carrying of both protein and conventional small molecule drugs without leakage of the drugs and denaturation of the proteins was developed. NPSCs were able to co-deliver the small molecule drug and protein into cell cytoplasm. [14] Interestingly, nanotechnology-based medicines are applicable for therapeutic, diagnostic and also for theranostic purposes/combination of treatment and diagnosis. The numerous advantages that nanomedicines possess, over current conventional treatments, have attracted pharmaceutical industry, resulting in the mass production and marketing of various anticancer nanomedicines. Nanotechnology is expected to play a vital role in future cancer pharmacotherapy.

The Effect of Physicochemical Properties in the Anticancer Activity of Nanomaterials

Nanomaterials possess unique physicochemical characteristics attributable to their ultrasmall size, high surface to volume ratio, hydrophilicity/ lipophilicity, composition, presence of surface biochemical agents, aggregation and physical appearance. ${ }^{[15]}$ The impact of the various physicochemical properties on the physiological interaction of anticancer nanoparticles and which affect their therapeutic efficacy, safety and diagnostic accuracy are discussed in this review. In an effort to develop biocompatible vectors for cancer gene therapy, chitosan-based nanocomplexes were synthesized and the transfection efficiency of the nanocomplexes for gene delivery to breast cancer cells was assessed. [16] Results suggested that, nanocarrier containing low molecular weight chitosans were not stable in the transfection medium and hence unsuitable for gene delivery. However, carriers which contain intermediate-DA (degree of acetylation) chitosans were efficient. On the other hand, carriers containing lowDA chitosans were too stable to release the miRNA cargo. Findings predict that higher-DA chitosans were able to release the miRNA into the cytosol more efficiently than low-DA (i.e., high charge density) chitosans because of the lower stability of the carriers. Negatively charged carriers (i.e., (+/-) charge ratios less than 1) were not suitable for transfection. Besides, it was observed that positively charged carriers are effective only when they exceed a certain concentration. It was concluded that, for optimal transfection, perfect balance of the molecular weight, DA and (+/-) charge ratios is required. These suggest that, physicochemical properties can affect the biological activity of anticancer nanomaterials and are critical to the clinical translation of candidate nanomedicines.

To ensure safe and effective in vivo administration of a material, understanding the underlying mechanism of the effect of the various physicochemical attributes of nanomaterials in their in vivo behavior and performance is a main prerequisite. ${ }^{[17]}$ Physicochemical 
properties of nanoparticles are probably dependent on environmental conditions. The changeable compositions and structures could cause nanomaterials to show dynamic changes in physiological conditions which results in the occurrence of special behaviors under biological environment. ${ }^{[18]}$ Therefore, for solid conclusions with regards to the relationship between the in vivo behavior of nanomaterials and their physicochemical properties, which is necessary in the development stage of materials used for clinical applications, accurate characterization of physicochemical properties of nanoparticles in a biological environment and knowledge of the interactions of nanomaterials with the biological molecules are needed. [19] This requires reliable and robust techniques to study the various physicochemical properties of nanomedicines. Furthermore, recent research on therapeutic and diagnostic applications of nanomaterials is identifying critical parameters for the material's compatibility with physiological systems. It has been discovered that apart from pyhsicochemical properties pathophysiology associated with tumor growth can be exploited for designing tumor targeting gold nanoparticles. [16] Therefore, a rationale characterization strategy for medicinal nanomaterials should include three essential parts [20], (1) physicochemical characterization of surface charge, size, surface area, size distribution, composition and shape etc., (2) in vitro assays such as protein adsorption and blood contact properties, and (3) in vivo studies including assessment of nanoparticles' effects on various biological systems and pharmacological and toxicological properties.

The challenges associated with nanomaterial physicochemical characterization have shifted over the last decades. [21] Initally, researchers grappled with proper ways to assess size, charge, or composition, including which measurement technique was most suited and what the most appropriate measurement conditions. Now it is well accepted that materials should be analyzed by multiple orthogonal analytical techniques and under the appropriate biologically relevant conditions. Many of the attributes analyzed to characterize conventional drugs apply to nanomaterials. Similar to larger materials, properties such as identity, solubility, molecular structure, boiling point, chemical composition, vapor pressure, melting point, partition coefficient, and dissociation constant should be determined for nanomaterials too. [17] However, the definition and analysis of these attributes can differ for nanomaterials. Some parameters including zeta potential, size or size range, shape, porosity, surface area, polydispersity, stability, surface functionalization and others are more importantly analyzed for nanomaterials. [17]

Size

Size and size range of nanoparticles are important attributes that influence many properties of nanoparticles, like cellular uptake, transport, tumor accumulation, capacity of drug carrying and release, distribution, targeting of site of action, therapeutic efficacy and toxicity. [22] Optimum nanoparicle size is a prerequisite for successful delivery of anticancer drugs. For instance, the size of each endocytotic portal, which has its own dynamics and size rules, governs the taken up of nanoparticles by the endocytosis-mediated internalization. Larger particles $(>\sim 200 \mathrm{~nm})$ can not escape the reticuloendothelial system (RES) and very small sized particles $(<\sim 10 \mathrm{~nm})$ are prone to removal by the kidney. ${ }^{[23]}$ Nanoparticles, owing to their small particle size, display improved pharmacokinetic profile and in vivo distribution of loaded drugs and thus decrease toxicity owing their specific accumulation at the intended site. ${ }^{[23]}$ It has been shown that specific distribution of drug in tumors can be enhanced by controlling the nanoparticle size. Smaller size nanoparticles $(39 \mathrm{~nm})$ were easily taken up by pancreatic carcinoma tumor cells and demonstrated higher efficacy than that of larger size nanoparticles (68 $\mathrm{nm}$ and $116 \mathrm{~nm}$ ). [24] Along these lines, a surface modified nanoparticle (PEGylated carbonate apatite (CA) designed to enhance the toxicity and delivery of anastrozole (hydrophobic) and gemcitabine (hydrophilic) to breast cancer cells was developed. Results evidenced that, the coated apatite/drug nanoparticles demonstrated higher toxicity and tumor regression activities compared to the free drug and uncoated apatite/drug nanoparticles. It was suggested that, the surface modification successfully gave rise to optimum particles size leading to more effective uptake and favorable pharmacokinetic properties of the nanoparticles. [25] Furthermore, some nanoparicle properties depend upon surface area which in turn depends upon particle size. Decreasing the size leads to an exponential increase in surface area of a particle, thus, particles having the same composition can have significant difference in levels of therapeutic and toxic potential depending on both particle size and surface reactivity. ${ }^{[26]}$ In a study aimed to determine whether differences in tumor pathophysiology (changes in tumor volume) has an impact on tumor accumulation and penetration of different size spherical gold nanoparticles (AuNPs), it was found that difference in pathophysiology related to tumor volume can result in selective change in tumor uptake of different size nanoparticles. [27] It was found that AuNPs having diameters of $15-45 \mathrm{~nm}$ are most suitable for tumors which have sizes greater than $1.0 \mathrm{~cm}^{3}$. It was also demonstrated that, while transport of bigger nanomaterials is mainly due to Brownian motion, for smaller nanoparticles, tumor accumulation depends on the frequency of interaction of particles with the perivascular extracellular matrix. Reduction of interstitial volume and enhanced porosity of stroma of tumors, render large AuNPs more effective. As the payloads of small sized AuNPs are less than larger 
particles oftentimes, they may not be ideal in many applications. Moreover, some therapeutic and diagnostic nanomaterials are not simple and consist of combinations of molecular self-assembly, encapsulation, and/or the use of nanosized metal or polymer cores, surfactants and/or proteins to improve solubility and perform special functions. It is necessary to characterize the precise size, size distribution and polydispersity index of such materials. ${ }^{[8]}$ Generally, particle size analysis can be broadly classified ed into two classes, imaging and light scattering. ${ }^{[28]}$ A sizing technique will be chosen depending on the required information. Light scattering methods include dynamic and static light scattering and laser diffraction (LD). The main imaging techniques are atomic force microscopy (AFM), scanning electron microscopy (SEM), and transmission electron microscopy (TEM). In order to cross-validate measurements and also acquire information about the polydispersity of a sample, size analysis should be done using two or more techniques. [28]

\section{Shape}

Nanotechnology-based materials fall into various groups of particles with a variety of characteristics which includes density, size, shape, and surface composition. [29] Based on their shape, nanomaterials are categorized as nanospheres, nanovesicles, nanoshells, nanotubes, nanohorns, nanofibers, nanowires, nanoribbons, nanorods, nanosticks, nanohelices, etc. In one study, development ofsurfacefunctionalized $\mathrm{Fe}_{3} \mathrm{O}_{4}$ magnetic nanoparticles of different shapes (spherical, hexagonal and wire-like) was carried out to investigate their in vitro and in vivo photothermal anticancer effect. $\mathrm{All} \mathrm{Fe}_{3} \mathrm{O}_{4}$ nanoparticles exhibited pronounced photothermal effects, triggered by red and near infrared laser irradiation. [30] Apart from size, nanoparticle shape may influence its biodistribution and membrane permeability. Four radioactive gold $(\mathrm{Au})$ nanostructures of varying shapes but equal size were synthesized and the distribution, tumor uptake, and intratumoral accumulation of the nanomaterials were quantitatively analyzed in murine EMT6 breast cancer model. Of the four shapes, the ${ }^{198} \mathrm{Au}$-incorporated nanospheres demonstrated the least removal by the reticuloendothelial system, best blood circulation, and greatest tumor uptake compared to nanodisks, nanorods, and nanocages. [31] Furthermore, while nanorods and nanocages reached the interior of the tumors, nanospheres and nanodisks were only able to reach on the tumor surfaces. The efficiency of drug retention at a tumor site can be enhanced by the ability of the delivery system to actively target its intended site or by improving vascular permeation due to the enhanced permeation and retention (EPR) effect. The efficiency of the EPR effect is governed by the physical and chemical properties of the nanomaterials, including size and shape. In a study using an in vitro microcirculation model, to assess the impact of critical physical parameters such as the particle shape, size, density and a nanoparticle's affinity towards the vessel walls, smaller-sized and oblate-shaped nanoparticles showed a desirable transport activity indicated by their higher movement rates towards the vessel walls in the microcirculation. [29] Spherical, tubular, plate-like, or nano-porous particles of similar components may differ significantly in their surface energy, biological activity, and access to various physiological structures, such as cell walls and capillary vessels. There are different electron microscopy methods including cryogenics, energy dispersive $\mathrm{X}$-ray spectroscopy, and threedimensional tomography that can give more information about a particle like shape, morphology, and composition. ${ }^{[28]}$ Methods such as AFM, SEM, and TEM are used to assess the shape distribution in a sample of a nanoparticle. Transmission electron microscope (TEM) gives images and chemical information of nanoparticles with better spatial resolution improving the morphological and structural assessment of nanomaterials at the atomic scale. The advantages of the unique nanopatricle characteristics in the treatment of cancer are shown in figure 2 .

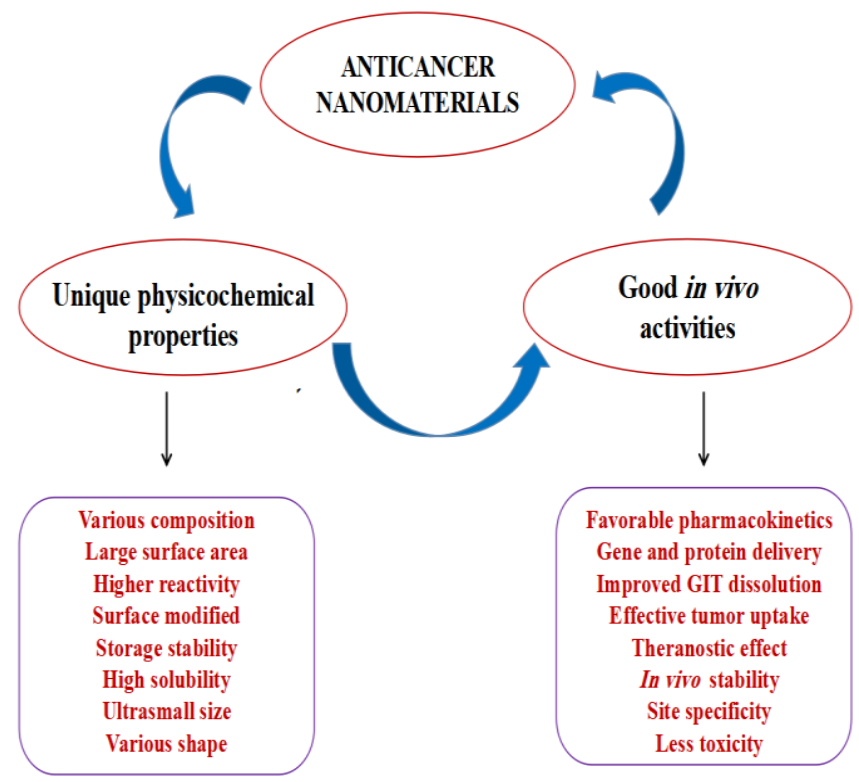

Fig. 2: The unique nanoparticle characteristics and their advantages in cancer treatment.

\section{Composition}

There are many varieties of nanomaterials for detection and/or imaging of cancer and site specific delivery of anticancer drugs. Nanomaterials are grouped into four classes [20]: (1) Organic nanoparticles (dendrimers, polymeric nanoparticles, functionalized fullerenes), (2) Inorganic nanoparticles (iron oxide core particles), (3) organic-inorganic hybrids (quantum dots), and (4) biological nanomaterials (liposomes). Each class has a distinctly different composition leading to varying physical characteristics, such as stability, solubility, surface properties, and functions. Besides, within a single group, possibly a variety of product compositions is available, each with special physical 
and chemical properties, and each group requires a different strategy for assessing its properties. [8] Nanoparticles can be designed and synthesized in a wide range of shapes, sizes, and components for unique and specific use. Recently folate conjugated nanomicelles loaded with curcumin-difluorinated (CDF), a curcumin analogue, targeted to retinoblastoma were synthesized. The drug loaded nanomicelles showed good performance, such as increased drug stability and high drug loading capacity and a sustained release profile, enhancing delivery of a large amount of CDF to the folate receptor over-expressing cancer cells. [32] The safety of the formulation was indicated by the absence of adverse toxicity on a retinal pigment epithelial cell (ARPE-19) of human. Other nanoparticulate carriers of various composition, including drug nanocrystals, nanoemulsions, and polyelectrolyte carriers were able to enhance drug solubility and control release kinetics of the drug in plasma and at the tumor. [33]

Polymeric nanoparticles represent effective carriers for drug delivery. In an investigation of internalization of tamoxifen citrate loaded polylactide-co-glycolide (PLGA) based nanoparticles (TNPs), the drug loaded nanoparticles showed greater cytotoxicity than the free drug and nanocarriers were taken up well by the MCF7 breast cancer cells, which was observed to be

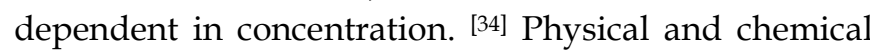
properties of a polymer, such as crystallinity, molecular weight, dispersity index, and hydrophobicity control degradation as well as drug release kinetics of polymeric nanomaterials. [35] Biological and physicochemical characterization of nanocomplexes intended for gene delivery to breast cancer cells demonstrated that, transfection efficiency depended on the properties of chitosan (polymer) highly. [16] Findings of the study anticipated that complexes of intermediate polymer molecular weight, positively charged, and of higher-DA (degree of acetylation) chitosans were able to release the miRNA into the cytosol more efficiently. In another study, a poly (D, Llactide-co-glycolide) (PLGA)-based formulation of miRNA-150 (miR-150-NF) was developed. [36] Incorporation of polyethyleneimine (a positively charged polymer), into the PLGA matrix, was carried out to enhance the encapsulation of miR-150. Characterization of miR-150-NF revealed that they had high encapsulation effciency $(\sim 78 \%)$ and also showed sustained release property.

Assessment of the intrinsic properties of inorganic materials for imaging and therapeutic purposes has been the focus of several studies. A multifunctional nanomaterial was contracted by covering magnetic iron oxide nanoclusters using a near-infrared lightabsorbing polymer polypyrrole (PPy), to obtain $\mathrm{Fe}_{3} \mathrm{O}_{4} @$ PPy core-shell nanoparticles, After functionalization with polyethylene glycol these nanoparticles were intended to be employed for imaging guided and remotely controlled cancer combination therapy. [37] The theranostic agent showed profound synergistic activity against tumor in biological environment. Moreover, surface characteristics and modification of gold nanoparticles have been employed for the administration of surfacebound anticancer drugs. In a research work, a strategy for direct synthesis of thiolated gold nanoparticles, using tri-sodium citrate reduction method, was demonstrated. A monodispersed and small sized gold nanoparticles $(\sim 20 \mathrm{~nm})$ were obtained and then conjugated with methotrexate. It was revealed that the methotrexate loaded gold nanoparticles caused higher toxicity on a breast cancer cell line (MCF-7) compared to the free drug and free gold nanoparticles. [38] However, compared to types of anticancer nanoparticles, inorganic nanoparticles may not be advantageous for systemic administration. Because they do not possess controlled release properties, are not biodegradable, and have low drug loading. [39] Dendrimers are globular molecules with branching structures and surface functional moieties available for modification. A dendronized heparine-doxorubicin (heparine-DOX) conjugate drug carrier sensitive to $\mathrm{pH}$ was prepared and characterized. The nanoparticle exhibited promising safety and high in vivo tumor inhibition as a result of the combined effects of polymer hybrid, dendrimer, and heparin nanoparticles. [40] The various physicochemical properties of anticancer nanomaterials and the physiological activities influenced by them are given in table 2 .

Table 2: Physicochemical characteristics of anticancer nanomaterials and the affected physiological activities.

\begin{tabular}{|c|c|}
\hline Parameter & Physiological activity \\
\hline Size & $\begin{array}{l}\text { Intratumor accumulation, cellular uptake, drug } \\
\text { loading and release, transport, distribution, tissue } \\
\text { targeting, toxicity, and therapeutic efficacy }\end{array}$ \\
\hline Shape & $\begin{array}{l}\text { Blood circulation, clearance by the } \\
\text { reticuloendothelial system, tumor uptake, } \\
\text { distribution, and absorption }\end{array}$ \\
\hline Com & $\begin{array}{l}\text { Transfection efficiency, release kinetics in the } \\
\text { blood and at the tumor site, cellular uptake, } \\
\text { cytotoxicity, and tumor targeting }\end{array}$ \\
\hline $\begin{array}{l}\text { Surface } \\
\text { properties }\end{array}$ & $\begin{array}{l}\text { Distribution, cytotoxicity, cellular uptake, } \\
\text { receptor mediated endocytosis, endosomal } \\
\text { entrapment, gastrointestinal dissolution, } \\
\text { distribution, and biocompatibility }\end{array}$ \\
\hline Stability & $\begin{array}{l}\text { Therapeutic efficacy, blood circulation time, } \\
\text { protein binding, and evasion of the immune } \\
\text { system }\end{array}$ \\
\hline $\begin{array}{l}\text { Drug loading } \\
\text { and release }\end{array}$ & Therapeutic efficacy and site specificity \\
\hline
\end{tabular}

Elemental analysis, frequently used to assess the purity of small molecule drugs, can be employed to analyze the composition and ratios of elements available in a sample of nanopreparation if an element has been used in the synthesis process. [41] For core-shell metal nanoparticles, the ratio of core to shell material ratios are analyzed. The composition of nanomaterials is analyzed using atomic emission (AE) and atomic absorption (AA) spectroscopies. [8] For imaging 
applications using iron oxide nanoparticles or gadolinium (Gd)-based chelates, composition analysis is necessary to assay metals available in the formulation. [8] Inductively coupled plasmon optical emission spectroscopy (ICP-OES) is a good alternative to quantify $\mathrm{Gd}$ in such contrast agents. Furthermore, since biomedical nanoformulations can be composed of a variety of substances, analysis of chemical composition is essential to ensure their purity and homogeneity. Purity analysis should consider the presence of free metals and chelates, solvents, dimers, unconjugated therapeutic or other agents, precursors. ${ }^{[8]}$ Surface properties

Unlike conventional pharmaceuticals, nanoparticles may be multipurpose systems with many components. [42] The outer surface of a nanoparticle may be attached to polymers to increase solubility or assist the particle escape the immune system, drugs, targeting moieties (like antibodies), and imaging contrast agents for diagnostic agents. In depth understanding of the surface is needed to achieve the desired safety and efficacy of a nanomaterial based formulation, because surface characteristics of a nanoparticle will impact its in vivo performance. [28] The common parameters in characterizing the nanoparticle surface are its charge, hydrophilic character, and targeting agents presence. [28] In a study, the mechanism and efficiency of endocytosis of MCM-4 type organically surface modified mesoporous silica nanoparticles (FITC-MSN) with various charges was examined on human cervical cancer cells (HeLa). Three functional groups, 3aminopropyl (AP), N-(2-aminoethyl)-3-aminopropyl (AEAP), and N-folate-3-aminopropyl (FAP), were attached onto the external surface of the FITC-MSN. The amino groups of the 3-aminopropyl and the N-(2aminoethyl)-3-aminopropyl were transformed into guanidinopropyl (GP) and 3-[N-(2guanidinoethyl)guanidino]propyl (GEGP) groups, respectively. Results revealed that the uptake of the nanoparticles can be regulated by varying the surface functional groups. The folate groups on FAP-MSN were also able to facilitate a receptor mediated endocytosis. The uptake of FAP-MSN was to some extent minimized in presence of folic acid, indicating that folic acid receptors on the surface of HeLa cell mediated its mechanism of endocytosis. It was revealed that the highly negatively charged FITC- and AP-MSNs were able to escape from endosomes, while positively charged particles, such as GP-, GEGP- and FAP-MSNs, were entrapped within endosomes. These result indicated that the surface functional groups have an impact on the ability of nanoparticles to escape endosomal entrapment, which is an important factor in designing intracellular drug delivery systems. [43]

Polymeric drug delivery vehicles can be tailored to release drugs stimulated by environmental conditions or external stimulus like, electricity, light, biomolecules, enzymes, and temperature. $\mathrm{pH}$ dependent solubility would significantly help in enhancing the oral bioavailability of drug. In a study aimed to improve the water solubility and the anticancer activity of camptothecin (CPT) for colon cancer treatment, CPT was incorporated into plymeric nanoparticles. ${ }^{[44]} \mathrm{A} \mathrm{pH}$ sensitive polymer coat was chosen to minimize the rapid dissolution of camptothecin in the gastrointestinal tract. It was found that camptothecin nanoparticles coated with a polymer, gave no release in the simulated gastric fluid, maximum release in the colonic area, and was hardly released in the simulated intestinal fluid. Similarly polyethylene glycolytion of a nanocomposite developed by covering magnetic iron oxide nanoclusters with a near-infrared light-absorbing polymer polypyrrole (PPy), resulted in a novel multipurpose theranostic nanomaterial applicable for cancer therapy. [37] In an effort to enhance the delivery and cytotoxicity of anastrozole (hydrophobic) and gemcitabine (hydrophilic) in breast cancer cells, researchers developed a surface modified nanoparticle (PEGylated carbonate apatite (CA). [25] The coated apatite/drug nanoparticles showed higher cytotoxicity and tumor regression activities compared to the uncoated apatite/drug nanoparticles and free drugs, which indicate that surface modification, resulted in optimum particles size leading to more effective tumor retention and favorable pharmacokinetics of the particles.

Zeta potential is commonly used as an indication of the actual surface charge of nanoparticles. Zeta potential measurement techniques are based on laser doppler velocimetry and light scattering. There are no straightforward ways of quantifying the density, distribution, and coating and targeting molecules presentation. [28] Hence, it is perceived that surface coating is uniform, or the targeting moiety is bound to the surface and able to bind to the intended receptor. Biological assays of nanoparticles in the presence and absence of targeting moieties are useful in getting information about targeting ligand accessibility and presentation. ${ }^{28]}$ A number of ways that assess desired target-site binding and protein interactions are present. Analytical methods such as nanopore-based detection through tunable resistive pulse sensing (TRPS), isothermal titration calorimetry (ITC), quartz crystal microbalance, archimedes, dual polarization interferometry (DPI), and surface plasmon resonance. [28] All the approaches have different principles of evaluating ligand binding. Quantification of surface conjugates is commonly done using RP-HPLC. [28] Mass spectrometry is also used for better sensitivity. When $\mathrm{UV}-\mathrm{Vis}$ detection is not sensitive enough, assay and detection of polymers is performed with ELSD or CAD. Stability

One property of nanoparticles that is necessary for application in drug delivery is that, stability with respect to size, surface morphology, size distribution and other important properties. Stability testing 
provides information on how the properties of a nanomaterial changes with time under the influence of different environmental factors. Some nanoparticles for drug delivery and/or diagnosis are multifunctional systems with complex composition tailored to have simultaneous targeting, therapeutic, and imaging activities. The stability of all components is important to maintain the intended therapeutic or diagnostic effect of the nanoparticles. [8] Premature release of any of the components from the multicomponent platform may render it ineffective. It is essential to assess the in vitro functional component stability under biological conditions. Characterization of $\mathrm{pH}$-sensitive amphiphilic dendron and linear polymer conjugate based nanoparticle as a drug delivery platform, dendronized heparin-doxorubicin (heparine-DOX) conjugate. ${ }^{[40]}$ It demonstrated a promising biosafety and high in vivo antitumor action of the nanoplatform. It was suggested, the observed results were as a result of combined effects of dendrimer, polymer hybrid and heparin indicating the stability of the nanoparticle components in the biological environment. The ability to escape or exploit the human natural immune defense system may dictate the success or failure of a nanoparticle-based drug. Blood stability can be achieved by developing strategies to decrease protein binding and escape the immune system. [45] The stability of a functional self-assembled protein coating (HFBII) on ${ }^{18} \mathrm{~F}-$ labeled hydrocarbonized porous silicon nanoparticles (THCPSi) was examined in vitro in biological conditions relevant for systemic administration. ${ }^{[45]}$ Results of the study indicated that circulating HFBII-18 $\mathrm{F}-\mathrm{THCPSi}$ nanoparticles retained their HFBII coating for hours after systemic administration, proving the feasibility of increasing the circulation time with the functionalization using biochemicals. Furthermore, a class of multiferroic nanoparticles, targeted magnetoelectric nanoparticles (MENs), was developed to control drug release and delivery. The in vitro and in vivo preclinical studies on nude mice with xenografed ovarian carcinomas revealed that, surface functionalization enabled the drug (paclitaxel (PTX)) to be attached to MENs and hence avoid its premature release. The externally controlled delivery/release mechanism provided flexibility in surface functionalization to efficiently attach the drug to the nanoparticles for avoiding considerable drug's loss in its journey, through the circulatory system, to the target site. [46]

Colloidal stability in an aqueous and physiological medium is one of the most important issues related to the biomedical applications of nanoparticles. ${ }^{[47]}$ It is also important to determine the stability of nanoparticles under environmental and processing conditions to account for the effects of short-term and long-term storage, lyophilization, ultrafiltration, thermal exposure, $\mathrm{pH}$ variation, freeze-thawing, and exposure to light. ${ }^{[8]}$ In order to exploit the anticancer effect of garcinol (GAR), a naturally occurring polyisoprenylated phenolic agent, a novel nanoparticle (GAR-NPs) was developed by entrapping garcinol in Poly(lactic-co-glycolic acid) or PLGA. [48] Long term stability analysis of the free flowing powder of GARNPs obtained by lyophilisation was carried out by assessing stored samples to determine particle size and charge on the surface. The particle size was slightly increased with a moderate change in polydispersity index (PDI) value, and zeta potential exhibited the least variation; this indicated the good storage stability of GAR-NPs. In a research to improve the aqueous solubility of camptothecin (CPT), hydrophobic antineoplastic agent, CPT loaded polymeric nanoparticles were synthesized and characterized. Stability study was performed to analyze the prepared plain and drug loaded polymeric nanoformulations ability to remain unchanged due to atmospheric/environmental changes. Prepared plain and camptothecin loaded polymeric nanoparticles demonstrated unremarkable change in average particle size, particle size uniformity, surface area, zeta potential, drug content and drug release after both long term and accelerated conditions storage conditions. ${ }^{[49]}$

\section{Drug loading}

Drug delivery is widely explored applications of pharmaceutical nanotechnology. A high drug loading capacity is the measure of successful nanoparticulate system because it minimizes the amount of matrix material to be administered. [50] Drug loading can be achieved using two approaches: (1) Incorporation method: where the drug is added during the formation of nanoparticle. It is a commonly used method for lipophilic drugs. (2) Adsorption method: where the drug is made to be adsorbed on nanoparticle. The formed nanoparticle is placed in concentrated solution of drug and adsorption proceeds. Total drug is commonly measured by RP-HPLC. Drug loading and drug release can possibly assayed by florescent tags. [28] In case the fluorescent tag is not included in the nanoformulation, this method is not encouraged.

\section{Drug release}

Drug release should be assessed, under relevant biological conditions, which accounts for the $\mathrm{pH}$, media, temperature, sampling time and volume. [28] In a study, synthesis and characterization of a modifed polyethyleneimine (PEI) coated magnetic nanoparticles, in which PEI was modifed by sebacoyl chloride and targeted by folic acid to obtained polymer (PEI-Sb-FA) was performed. The obtained PEI-Sb-FA was then coated on magnetic nanoparticles (MNPs) to stabilize them and curcumin was finally loaded on PEI-Sb-FAMNP to study the release behavior in different $\mathrm{pH}$. The released drug in acidic $\mathrm{pH}=4.5$ was more than $\mathrm{pH}=$ 7.4, showing drug release sensitivity toward $\mathrm{pH}$ of media. [51]

In polymer coated nanoparticles the release is affected by movement of drug from core across the polymeric membrane. Many techniques can be employed to 
control drug release and most release methods require separation of the drug and its carrier. [52] Equilibrium dialysis, ultracentrifugation, diffusion cells, and ultrafiltration are some of the most commonly used approaches. A method is chosen depending on individual preparation and the ease of separation of the released drug from the nanoparticle. For drugs attached to a polymeric skeleton, assessment of drug release in blood is important. [28] It is essential to simulate physiological environment to control $\mathrm{pH}$ mediated or enzymatic separation of the drug from the backbone. The separation site as well as the activity of the separated drug must also be studied typically by RP-HPLC.

\section{Future Perspectives}

Although there have been improvements in cancer treatment, cancer is still quite difficult to treat owing to lack of safety and effectiveness of conventional anticancer drugs. The application of nanotechnology in cancer treatment and/ diagnosis is perceived to improve the therapeutic outcomes. Identifying precisely what qualifies a nanomedicine or a theranostic is difficult, however, a rational formulation design and development, based on standard criteria for acceptable quality, safety, efficacy, and desirable pharmaceutical characteristics, is essential. This review discusses the role of physical and chemical properties on the therapeutic efficacy of nanoparticles found to effective for cancer treatment and/or diagnosis in the past decade. Most efforts to improve cancer diagnosis and treatment through nanotechnology are at the research or development stage. There are no common standardized guidelines used to study the different nanoparicle attributes. Moreover, the findings on the impact of physical and chemical attributes of nanomaterials on their therapeutic efficacy apply to the specific materials tested. To achieve fast and successful clinical translation of nanotechnology-based anticancer drug products, complete characterization in order to support highly reproducible manufacturing processes is another important prerequisite. Common standardized methodologies or regulatory guidelines are required to study the different attributes of nanoparticles developed for diagnostic or therapeutic use. Generalized principles help to establish in vivo/ in vitro correlation between physicochemical characteristics and in vivo performance. If this can be successfully achieved, it has the benefit of reducing development cost and time. To help develop such generalized principles, collaboration among researchers is important in pursuing preclinical physicochemical characterization. Although the use of nanotechnology for detection and treatment of cancer is mainly in the development stage, already a number of nanoplatforms-based drugs are commercialized and many are in clinical trials. It is expected that nanotechnology has great potential in improving future cancer treatment.

\section{REFERENCES}

1. Emeje MO, Obidike IC, Akpabio EI, Ofoefule SI. Nanotechnology in Drug Delivery. In: Recent Advances in Novel Drug Carrier Systems, Sezer, A.D. (Ed.). Chapter 4, InTech Publisher, Rijeka, 2012, pp. 69-106.

2. Laouini A, Jaafer-Maalej C, Limayem-Blouza I, Sfar S, Charcosset C, Fessi H. Preparation, characterization and applications of liposomes: state of the art. Journal of colloid Science and Biotechnology. 2012; 1(2):147-168.

3. Gad SC. Pharmaceutical manufacturing handbook: production and processes. John Wiley \& Sons, 2008.

4. Zhu $M$, Nie G, Meng $H$, Xia T, Nel A, Zhao Y. Physicochemical properties determine nanomaterial cellular uptake, transport, and fate. Acc. Chem. Res. 2012; 46(3):622 631.

5. Zhang J, Tang H, Liu Z, Chen B. Effects of major parameters of nanoparticles on their physical and chemical properties and recent application of nanodrug delivery system in targeted chemotherapy. Int. J. Nanomed. 2017; 12:8483.

6. Zhang L, Gu FX, Chan JM, Wang AZ, Langer RS, Farokhzad OC. Nanoparticles in medicine: therapeutic applications and developments. Clin pharmacol Ther. 2008; 83(5):761-769.

7. Markeb AA, El-Maali NA, Sayed DM, Osama A, AbdelMalek MAY, Zaki AH, Elwanis MEA, Driscoll JJ. Synthesis, structural characterization, and preclinical efficacy of a novel paclitaxel-loaded alginate nanoparticle for breast cancer treatment. Int J Breast Cancer. 2016; 2016.

8. Amiji MM. Nanotechnology for cancer therapy. CRC press, 2006.

9. Guerrini L, Alvarez-Puebla R, Pazos-Perez N. Surface modifications of nanoparticles for stability in biological fluids. Materials. 2018; 11(7):1154.

10. Murugan C, Rayappan K, Thangam R, Bhanumathi R, Shanthi K, Vivek R, Thirumurugan R, Bhattacharyya A, Sivasubramanian S, Gunasekaran P, Kannan S. Combinatorial nanocarrier based drug delivery approach for amalgamation of anti-tumor agents in breast cancer cells: an improved nanomedicine strategy. Sci Rep. 2016; 6:34053.

11. Liu L, Ye Q, Lu M, Lo Y-C, Hsu Y-H, Wei M-C, Chen Y-H, Lo S-C, Wang S-J, Bain DJ, Ho C. A new approach to reduce toxicities and to improve bioavailabilities of platinumcontaining anti-cancer nanodrugs. Sci Rep. 2015; 5:10881.

12. Zhang $Y$, Li R-J, Ying $X$, Tian W, Yao H-J, Men $Y, Y u ~ Y$, Zhang L, Ju R-J, Wang X-X, Zhou J, Chen J-X, Li N, Lu W-L. Targeting therapy with mitosomal daunorubicin plus amlodipine has the potential to circumvent intrinsic resistant breast cancer. Mol. Pharm. 2010; 8(1):162-175.

13. Liu Y,Yang M, Zhang J, Zhi X, Li C, Zhang C, Pan F, Wang K, Yang Y, Fuentea JM, Cui D. Human induced pluripotent stem cells for tumor targeted delivery of gold nanorods and enhanced photothermal therapy. ACS Nano. 2016; 10(2):23752385.

14. Kim CS, Mout R, Zhao Y, Yeh Y-C, Tang R, Jeong Y, Duncan B, Hardy JA, Rotello VM. Co-delivery of protein and small molecule therapeutics using nanoparticle-stabilized nanocapsules. Bioconjugate chem. 2015; 26(5):950-954.

15. Navya P, Daima HK. Rational engineering of physicochemical properties of nanomaterials for biomedical applications with nanotoxicological perspectives. Nano Converg. 2016; 3(1):1.

16. Santos-Carballal B, Aaldering L.J, Ritzefeld M, Pereira S, Sewald N, Moerschbacher BM, Götte M, Goycoolea FM. Physicochemical and biological characterization of chitosanmicroRNA nanocomplexes for gene delivery to MCF-7 breast cancer cells. Sci Rep 2015; 5:13567.

17. Lin P-C, Lina S, Wanga PC, Sridhar R. Techniques for physicochemical characterization of nanomaterials. Biotechnol Adv. 2014; 32(4):711-726.

18. Zhang X-Q, Xu X, Bertrand N, Pridgen E, Swamia A, Farokhzada OC. Interactions of nanomaterials and biological systems: Implications to personalized nanomedicine. Adv Drug Deliv Revi. 2012; 64(13):1363-1384. 
19. Boverhof DR, David RM. Nanomaterial characterization: considerations and needs for hazard assessment and safety evaluation. Anal. Bioanal. Chem. 2010; 396(3):953-961.

20. Patri AK, Dobrovolskaia MA, Stern ST, McNeil SE. Nanotechnology for cancer therapy. CRC Press, 2006, pp. 120-152.

21. Desai N. Challenges in development of nanoparticle-based therapeutics. AAPS J. 2012; 14(2):282-295.

22. Kumar D, Meenan BJ, Mutreja I, D`sa R, Dixon D. Controlling the size and size distribution of gold nanoparticles: a design of experiment study. Int J Nanosci. 2012; 11(02):1250023.

23. Hoshyar N, Gray S, Han H, Bao G. The effect of nanoparticle size on in vivo pharmacokinetics and cellular interaction. Nanomedicine, 2016; 11(6):673-692.

24. Zhao P, Zheng M, Yue C, Luo Z, Gong P, Gao G, Sheng Z, Zheng $C$, Cai L. Improving drug accumulation and photothermal efficacy in tumor depending on size of ICG loaded lipid-polymer nanoparticles. Biomaterials. 2014; 35(23):6037-6046.

25. Mozar FS, Chowdhury EH, Surface-modification of carbonate apatite nanoparticles enhances delivery and cytotoxicity of gemcitabine and anastrozole in breast cancer cells. Pharmaceutics. 2017; 9(2):21.

26. Khan IK, Saeed, Khan I. Nanoparticles: Properties, applications and toxicities. Arabian Journal of Chemistry, 2017.

27. Sykes EA, Dai Q, Sarsons CD, Chen J, Rocheleau JV, Hwang DM, Zheng G, Cramb DT, Rinker KD, Chan WC. Tailoring nanoparticle designs to target cancer based on tumor pathophysiology. PNAS. 2016; 113(9):201521265.

28. Christine V, Ponchel G, Polymer nanoparticles for nanomedicines. A guide for their design. Anticancer Res. 2017; 37:1544.

29. Toy R, Hyden E, Shoup C, Baskaran H, Karathanasis E. The effects of particle size, density and shape on margination of nanoparticles in microcirculation. Nanotechnology. 2011; 22(11):115101.

30. Chu M, Shao Y, Peng J, Dai X, Li H, Wu Q, Shi D. Nearinfrared laser light mediated cancer therapy by photothermal effect of Fe3O4 magnetic nanoparticles. Biomaterials. 2013; 34(16):4078-4088.

31. Black KC, Wang $Y$, Luehmann HP, Cai X, Xing W, Pang B, Zhao Y, Cutler CS, Wang LV, Liu Y, Xia Y. Radioactive 198Au-doped nanostructures with different shapes for in vivo analyses of their biodistribution, tumor uptake, and intratumoral distribution. ACS Nano. 2014; 8(5):4385-4394.

32. Alsaab H, Alzhrani R, Kesharwani P, Sau S, Boddu S, Lyer A. Folate decorated nanomicelles loaded with a potent curcumin analogue for targeting retinoblastoma. Pharmaceutics. 2017 9(2):15.

33. Lee JH, Yeo Y. Controlled drug release from pharmaceutical nanocarriers. Chemical engineering science. 2015; 125:75-84.

34. Maji R, Dey NS, Satapathy BS, Mukherjee B, Mondal S. Preparation and characterization of Tamoxifen citrate loaded nanoparticles for breast cancer therapy. Int J Nanomedicine. 2014; 9:3107.

35. Alexis F, Pridgen EM, Langer R, Faronkhzad OC. Drug delivery. Springer, Berlin, 2010, pp. 55-86.

36. Arora S, Swaminathan SK, Kirtane A, Srivastava SK, Bhardwaj A, Singh S, Panyam J, Singh AP. Synthesis, characterization, and evaluation of poly (D, L-lactide-coglycolide)-based nanoformulation of miRNA-150: potential implications for pancreatic cancer therapy. Int J Nanomedicine. 2014; 9:2933.

37. Wang C, Xu H, Liang C, Liu Y, Li Z, Yang G, Cheng L, Li Y, Liu Z. Iron oxide@ polypyrrole nanoparticles as a multifunctional drug carrier for remotely controlled cancer therapy with synergistic antitumor effect. ACS Nano, 2013; 7(8):6782-6795.

38. Mohamed AI, Hussain AK, Zayed GM, Shaykoon SM, Mahmoud RA. Preparation and Characterization of Cytotoxic DrugLoaded Gold Nanoparticles. Ijppr. 2016; 6(4):640-652.

39. Kirubakaran S, Thiruvenkatam V. Research on Diverse Applications of Nanotechnology in Biomedicine, Chemistry and Engineering. IGI Global, 2015.

40. She W, Li N, Luo K, Guo C, wang G, Geng Y, Gu Z. Dendronized heparin- doxorubicin conjugate based nanoparticle as $\mathrm{pH}$-responsive drug delivery system for cancer therapy. Biomaterials. 2013; 34(9):2252-2264.

41. Nash MA, Lai JJ, Hoffman AS, Yager P, Stayton PS. "Smart" diblock copolymers as templates for magnetic-core gold-shell nanoparticle synthesis. Nano Lett. 2009; 10(1):85-91.

42. Misra P. Applied Spectroscopy and the Science of nanomaterials. Springer, 2014.

43. Slowing I, Trewyn BG, Lin VS-Y. Effect of surface functionalization of MCM-41-type mesoporous silica nanoparticles on the endocytosis by human cancer cells. J. Am. Chem. Soc. 2006; 128(46):14792-14793.

44. Mahalingam M, Krishnamoorthy K. Camptothecin loaded poly (methacyclic acid-Co-methyl-methyacrylate) nanoparticles: Fabrication, characterization and cytotoxicity studies. Int J Pharm Pharm Sci. 2015; 7(10):135-140.

45. Conde J, Tian F, de la Fuente JM, Baptista PV. Cancer Nanotheranostics: What Have We Learned So Far? Front Chem. 2016; 3:71.

46. Rodzinski A, Guduru R, Liang P, Hadjikhani A, Stewart T, Stimphil E, Runowicz C, Cote R, Altman N, Datar R, Khizroev S. Targeted and controlled anticancer drug delivery and release with magnetoelectric nanoparticles. Sci Rep. 2016; 6:20867.

47. Moore TL, Rodriguez-Lorenzo L, Hirsch V, Balog S, Urban D, Jud C, Rothen-Rutishauser B, Lattuada M, Petri-Fink A. Nanoparticle colloidal stability in cell culture media and impact on cellular interactions. Chem Soc Rev. 2015; 44(17):6287-6305.

48. Gaonkar RH, Ganguly S, Dewanjee S, Sinha S, Gupta A, Ganguly S, Chattopadhyay D, Debnath MC. Garcinol loaded vitamin E TPGS emulsified PLGA nanoparticles: preparation, physicochemical characterization, in vitro and in vivo studies. Sc Rep. 2017; 7(1):530

49. Mahalingam M, Krishnamoorthy K. Fabrication, physicochemical characterization and evaluation of in vitro anticancer efficacy of a novel $\mathrm{pH}$ sensitive polymeric nanoparticles for efficient delivery of hydrophobic drug against colon cancer. J Appl Pharm Sci. 2015; 5(11):135-145.

50. Garg A, Visht S, Sharma PK, Kumar N. Formulation, characterization and application on nanoparticle: a review. Der Pharmacia Sin. 2011; 2(2):17-26.

51. Arabi S, Javar HA, Khoobi M. Preparation and Characterization of Modified Polyethyleneimine Magnetic Nanoparticles for Cancer Drug Delivery. J Nanomater. 2016; 2016.

52. Bhatia S. Natural polymer drug delivery systems. Springer, Cham, 2016, pp. 33-93.

HOW TO CITE THIS ARTICLE: Filli MS, Ibrahim AA, Aquib M, Abbas AB, Morshed A, Boakye-Yiadom KO, Kesse S, Farooq MA, Bazezy M, Wang BO. The Impact of Physicochemical Characteristics on Therapeutic Efficacy of Anticancer Nanomaterials: A Review. Int. J. Pharm. Sci. Drug Res. 2019; 11(2): 61-70. DOI: 10.25004/IJPSDR.2019.110203 